\title{
PD-L1 testing and immunotherapy selection - early laboratory experience and its potential role in head and neck cancer management
}

\author{
Andrei Bancu ${ }^{*, 1}$, Richard Cowan², Anshuman Chaturvedi ${ }^{3}$
}

1'Department of Histopathology and Molecular Pathology, St James's University Hospital, Leeds, UK; 2Department of Clinical Oncology, The Christie Hospital, Manchester, UK; ${ }^{3}$ Department of Histopathology, The Christie Hospital, Manchester, UK

\begin{abstract}
Anti-programmed cell death protein-1 (PD-1) therapy has been relatively recently approved in a defined context by NICE in adults in the management of recurrent and metastatic head and neck squamous cell carcinomas (HNSCC). In this context, companion diagnostic programmed cell death ligand-1 (PD-L1) testing, previously established at our center for lung and bladder tumors, was undertaken in a few head and neck cancer cases. The scope of this study was to audit the relevant PD-L1 data and integrate the findings in our current clinical practice, with a view to promote improved routine laboratory biomarkers in HNSCC. Histopathology reports documenting tumor type, PD-L1 result and type of clone/assay were included in this study. Over a 5-year period, PD-L1 testing was undertaken in 199 cancer cases, including 3 with head and neck squamous carcinoma with low focal positive staining. Immunotherapy treatment in HNSCC demonstrates a discreet but still significant improvement in the overall survival of PD-L1 positive subjects.
\end{abstract}

Keywords: PD-L1/PD-1; biomarkers; tumor proportion score; combined positive score; immunotherapy; head and neck squamous cell carcinomas

\section{Introduction}

Head and neck squamous cell carcinoma (HNSCC) is the $6^{\text {th }}$ most common type of cancer worldwide with an incidence that is expected to rise by more than one million new cases per year in the next 10 years. HNSCC originates in the squamous epithelium of the nasopharynx, nasal sinus, oropharynx, hypopharynx, larynx, and oral cavity mucosae and bears a poor prognosis that has only seen a mild improvement over the past 3 decades,

Received: April 2021; Accepted after review: June 2021; Published: June 2021.

${ }^{*}$ Corresponding author: Andrei Bancu, Department of Histopathology and Molecular Pathology, St James's University Hospital, Beckett St, Harehills, Leeds LS9 7TF, United Kingdom

Email: andrei.bancu92@gmail.com with $66 \%$ of patients being alive five years after diagnosis [1]. The prognosis is even direr considering the fact that more than $60 \%$ of patients are diagnosed with a disease that is locally advanced on presentation [2]. Standard treatment for locally advanced HNSCC includes radical excision of the primary tumor (including lymph node dissection as required) or definitive concurrent chemoradiation. Unfortunately, up to $60 \%$ of these treated patients will develop loco-regional recurrence within 2 years whilst $20-30 \%$ of them will develop distant metastases [3] therefore, further improvement in the clinical outcome of these patients is highly warranted by means of exploring molecular markers which might add prognostic value or act as companion diagnostic tools.

Immunotherapy with immune checkpoint inhibitors is a treatment method based on the 
blockade of inhibitory immune checkpoint pathways in order to restart the immune reaction against cancer cells [4]. Therefore, programmed cell death ligand-1 (PD-L1) expression represents an important biomarker and therapeutic target using this novel checkpoint inhibitor cancer immunotherapy.

Management for recurrent and metastatic HNSCC has been subjected to recent changes with NICE recommending (as of November 2017) the anti-programmed cell death protein1 (anti-PD-1) therapy in adults whose disease had progressed on platinum-based chemotherapy. Treatment with Nivolumab in this subset of patients improves overall survival by 2.6 months compared with docetaxel, methotrexate or cetuximab, although longer-term survival benefit, after 2 years, remains uncertain. As a result, lifeextending palliative treatment with Nivolumab has therefore been considered for all patients regardless of PD-L1 expression. However, it is still uncertain as whether this treatment provides a significant benefit for longer-term survival in tumor expressing less than 1\% PD$\mathrm{L} 1$ and further research is warranted in this direction [5].

Following successful trials in the United States with more recent FDA approval, immunotherapy with Pembrolizumab has started to become recommended (as of November 2020) by NICE, as an option for untreated metastatic or unresectable recurrent head and neck squamous cell carcinoma (HNSCC) in adults whose tumors express PDL1 with a combined positive score (CPS) of 1 or more. The committee also stated that monotherapy with Pembrolizumab should be used in metastatic/unresectable HNSCC regardless of their anatomic origin, inside or outside the oral cavity. In the former scenario, Pembrolizumab monotherapy is more effective than chemotherapy alone whilst in the latter sites (outside the oral cavity) this treatment is equally effective as cetuximab combination therapy but the overall costs are lower [6].

\section{Aim}

This study therefore aims to record data as per NICE recommendations and share it with a view to highlighting the case for improved routine laboratory biomarkers in HNSCC.

\section{Material and methods}

A retrospective study commenced between January 2014 and October 2019 at the Christie Hospital, Manchester, yielded a total of 199 PDL-1 tests performed on biopsies or resections showing a variety of carcinomas. Most of the analysis was performed on nonsmall cell carcinomas of the lung and urothelial carcinomas of the genitourinary tract. Among these cases, there were 3 requests for patients who were diagnosed and managed for progressive head and neck squamous cell carcinoma. Histopathology reports stating PDL1 analysis were identified, included and analyzed for the study. Assessment of PD-L1 membrane expression demonstrated staining, by clone SP263 (VENTANA PD-L1 SP263 Assay - Roche Diagnostics), across a range of tumor proportion scores (TPS) between 1$100 \%$. The specimens were considered to have PD-L1 expression if TPS $\geq 1 \%$. Two specimens were also assessed with the CPS, in which a cutoff $\geq 1$ would indicate positive PD-L1 expression.

\section{Results}

The PD-L1 tests were performed on three head and neck surgical excision/biopsy specimens who were previously reported as follows: The first case was a poorly differentiated invasive squamous cell carcinoma (SCC) of the tongue, the second case was a moderately differentiated invasive SCC of the retromolar region and the third case was diagnosed as a poorly differentiated invasive SCC of the uvula.

PD-L1 immunohistochemistry showed focal positive tumor staining in all three cases with tumor proportion score ranging from 1\% in the first two cases to $15 \%$ in the last case. The combined positive score was also calculated for the first two cases and this was scored as 2. The pattern of staining particularly involved the cells at the advancing 
edge of the tumor along with occasional positive staining within the tumor infiltrating lymphocytes and interspersed macrophages in the tumor microenvironment (Figure 1).
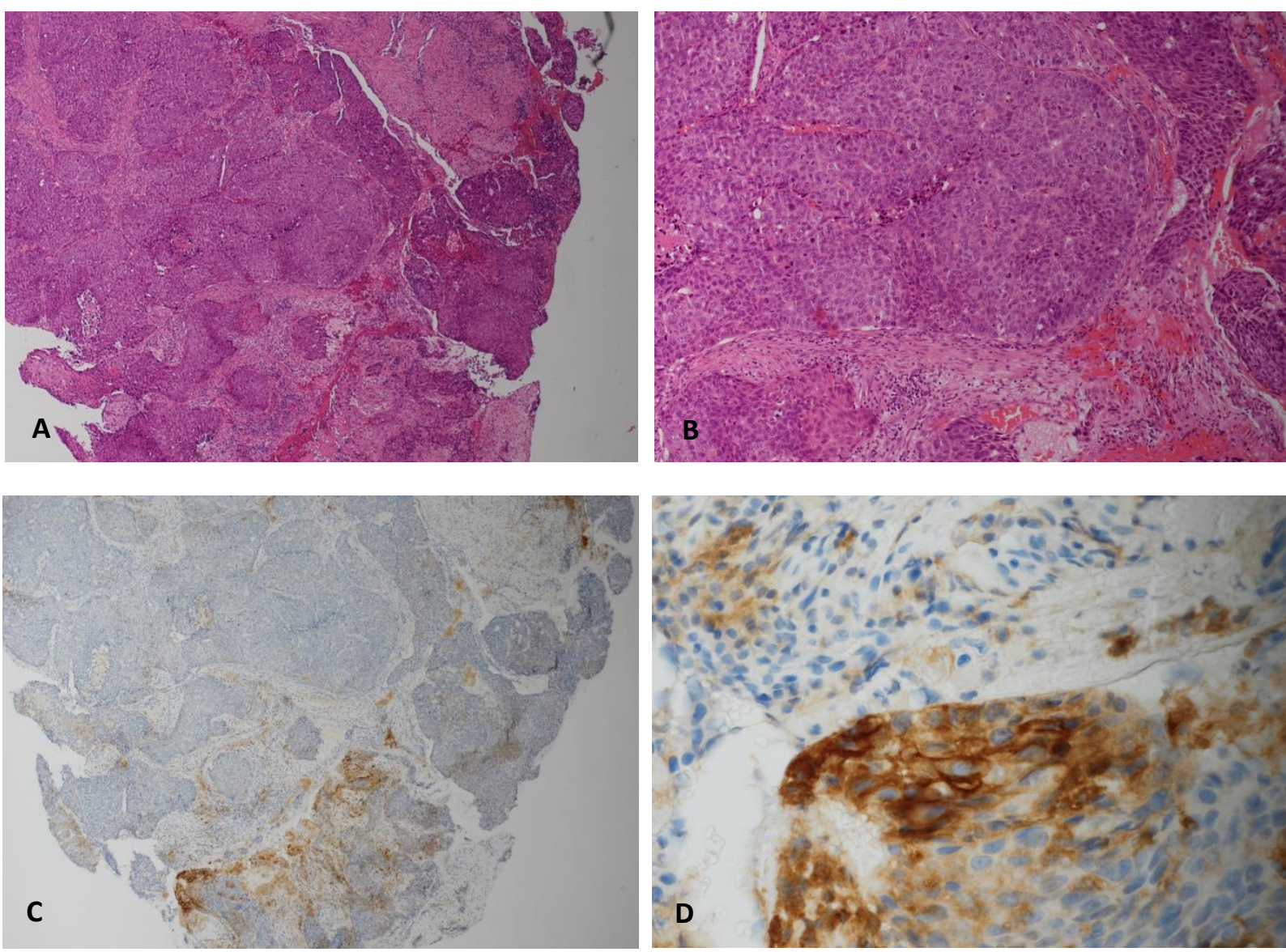

Fig. 1. An example of invasive poorly differentiated squamous cell carcinoma with basaloid morphology arising in in the left tongue $(A)$ and $(B)(H E, \times 40$ and $\times 100$, respectively). Focal positive expression of PD-L1 (TPS $=1 \%$; CPS=2) [IHC, anti-PD-L1 Ab, clone SP263, $\times 40$ (C) and $\times 400$ (D) assay on Ventana platform].

\section{Discussion}

The two patients showing 1\% PD-L1 expression were started on Nivolumab immune checkpoint inhibitor therapy following tumor recurrence after initial concurrent chemoradiotherapy. One patient managed to survive 4 months whilst the other 7 months, having his regimen stopped prematurely after 5 months due to persistence of side-effects. A third patient, who showed the highest PD-L1 expression (15\%), passed away 2 months following diagnosis whilst he was still on first line chemoradiotherapy.

Head and neck squamous cell carcinomas are well-known for their biological diversity and immune-suppressive nature, with immunetherapy treatment showing a discreet but significant improvement in overall survival rate.
Clinical trial evidence quoted by NICE, shows that Nivolumab improves overall survival by 2.6 months when compared to alternative 2nd line regimens. Although both patients with minimal PD-L1 expression have survived past this limit, the third one with a superior PD-L1 expression has passed away before initiating immunotherapy. These findings could be explained by the fact that a higher PD-L1 expression is associated with more aggressive tumor phenotypes directly impacting long-term survival. Additionally, negative cases or those with very low PD-L1 expression were still shown to respond to treatment [7, 8]. This outcome might be explained by the potential role of non-tumor cells such as T-cells, natural killer (NK) cells, tumor-associated macrophages (TAMs) and cancer-associated fibroblasts (CAFs) playing a part in PD-L1 
expression and further research is warranted in this direction to assess their potential functions and roles [9].

Although these results appear promising, genomic barriers such as tumor inter and intra heterogeneity still remain in front of PD-L1 being used as an effective biomarker in this neoplasm. Further research and development of other biomarkers such as tumor mutational burden are noted with a potential impact on centers which aim to perform PD-L1 testing in the nearby future. The more recent results of KEYNOTE-048 study published in October 2019 have recommended Pembrolizumab monotherapy as an appropriate first-line treatment for PD-L1-positive recurrent or metastatic HNSCC [10]. Immunotherapy with this checkpoint inhibitor is seen as optimal for these patients as PD-L1 expression is quantified by the CPS instead. When compared to TPS, the CPS takes into account not only the tumor cells but lymphocytes and macrophages as well against the total number of viable tumor cells which makes the tumor more sensitive to immunotherapy leading in turn to better survival. Any variation in staining between the different clones used in laboratories (e.g., SP263, DAKO 22C3, DAKO 28-8) will require further assessment, especially in the context of metastatic/recurrent HNSCC cases where more clones/assays are predicted to enter the market in the nearby future.

It is therefore anticipated that the requests for this molecular test will greatly increase in the upcoming months and years to follow,

\section{References}

1. Pulte $D$, Brenner $H$. Changes in survival in head and neck cancers in the late 20th and early $21^{\text {st }}$ century: a period analysis. Oncologist 2010; 15(9):994-1001. doi: 10.1634/theoncologist.2009-0289.

2. Cadoni G, Giraldi L, Petrelli L, et al. Prognostic factors in head and neck cancer: a 10-year retrospective analysis in a single-institution in Italy. Fattori prognostici del tumore testa-collo: un'analisi retrospettiva monocentrica di 10 anni. Acta Otorhinolaryngol Ital 2017; 37(6):458-466. doi:10.14639/0392-100X-1246

3. Denaro N, Merlano MC, Russi EG. Follow-up in head and neck cancer: do more does it mean putting more pressure on pathology workforce and laboratories worldwide.

\section{Conclusion}

Our study highlights an early increasing trend in PD-L1 tests performed in cases of metastatic/recurrent HNSCC with positive results anticipating a more favorable patient outcome when immune checkpoint inhibitor therapy was established.

Expression of PD-L1 by tumor cells generally correlates with improved outcome in patients suffering from recurrent or metastatic HNSCC who underwent treatment with antiPD-1/PD-L1 immunotherapy [4].

This test remains paramount in assessing tumor response to anti-PD-L1 therapy as further data about the correlation between expression and response in recurrent/metastatic head and neck squamous cell carcinomas await to be published. Other checkpoint inhibitors including durvalumab, avelumab, atezolizumab, tremelimumab and ipilumumab are actively being explored in clinical trials and await further validation [11]. Furthermore, any results could be altered by the fact that PD-L1 immunohistochemical assays were shown to vary in terms of positive samples with clone SP263 (the assay used in our study) staining a higher percentage of cells when compared to other clones.

\section{Competing interests}

The authors declare that they have no competing interests. do better? A systematic review and our proposal based on our experience. Clin Exp Otorhinolaryngol 2016; 9(4):287-297. doi:10.21053/ceo.2015.00976

4. Cohen EEW, Bell RB, Bifulco CB, et al. The Society for Immunotherapy of Cancer consensus statement on immunotherapy for the treatment of squamous cell carcinoma of the head and neck (HNSCC). J Immunother Cancer 2019; 7(1):184. doi: 10.1186/s40425019-0662-5.

5. National Institute for Health and Care Excellence. (2018). Nivolumab for treating squamous cell carcinoma of the head and neck 
after platinum-based chemotherapy. [https://www.nice.org.uk/guidance/ta490 available at 06.27.2021]

6. National Institute for Health and Care Excellence. (2020). Pembrolizumab for untreated metastatic or unresectable recurrent head and neck squamous cell carcinoma. [https://www.nice.org.uk/guidance/ta661 available at 06.27.2021]

7. Kim HS, Lee JY, Lim SH, et al. Association between PD-L1 and HPV status and the prognostic value of PD-L1 in oropharyngeal squamous cell carcinoma. Cancer Res Treat 2016; 48(2):527-536. doi: 10.4143/crt.2015.249

8. Yang WF, Wong MCM, Thomson PJ, Li KY, Su $Y X$. The prognostic role of PD-L1 expression for survival in head and neck squamous cell carcinoma: a systematic review and metaanalysis. Oral Oncol 2018; 86:81-90. doi: 10.1016/j.oraloncology.2018.09.016
9. Qiao XW, Jiang J, Pang X, et al. The Evolving landscape of PD-1/PD-L1 pathway in head and neck cancer. Front Immunol 2020; 11:1721. doi:10.3389/fimmu.2020.01721

10. Burtness B, Harrington KJ, Greil R, et al.; KEYNOTE-048 Investigators. Pembrolizumab alone or with chemotherapy versus cetuximab with chemotherapy for recurrent or metastatic squamous cell carcinoma of the head and neck (KEYNOTE-048): a randomised, open-label, phase 3 study. Lancet 2019; 394(10212):19151928. doi: 10.1016/S0140-6736(19)32591-7. Erratum in: Lancet. 2020; 395(10220):272.

11. Quenelle NB, Costantini CL. Immunotherapy in head and neck tumors: new options in advanced disease and beyond. J Unexplored Med Data 2018; 3:7. http://dx.doi.org/10.20517/2572-8180.2018.02 